\title{
Bilateral femoral head osteonecrosis in a patient with metastatic breast cancer receiving long-term zoledronic acid treatment: A case report
}

\author{
KAZUO MATSUURA ${ }^{1}$, TOSHIAKI SAEKI ${ }^{1}$, TAKAO TAKAHASHI ${ }^{2}$, TOMOAKI TORIGOE ${ }^{3}$, \\ KEISUKE WATARAI ${ }^{4}$, AKIHIKO OSAKI $^{1}$ and TAKASHI HOJYO ${ }^{1}$ \\ Departments of ${ }^{1}$ Breast Oncology, ${ }^{2}$ Palliative Medicine and ${ }^{3}$ Orthopaedic Oncology and Surgery, \\ Saitama Medical University International Medical Center, Hidaka, Saitama 350-1298; \\ ${ }^{4}$ Department of Orthopedic Surgery, Saitama Medical University Hospital, Hidaka, Saitama 350-0495, Japan
}

Received August 22, 2020; Accepted April 1, 2021

DOI: $10.3892 /$ mco.2021.2328

\begin{abstract}
Bone-modifying agents (BMAs), including bisphosphonate and anti-receptor activator of $\mathrm{NF}-\kappa \mathrm{B}$ ligand (RANKL) antibodies, are effective in treating bone metastases. The present study is a case report on the efficacy and side effects of long-term treatment with zoledronic acid, a BMA, in a 57-year-old woman. The patient was diagnosed with concurrent stage IV triple-negative breast cancer and stage II colon cancer. The patient experienced complete remission of both these cancers following chemotherapy, zoledronic acid treatment and irradiation for breast cancer and surgery for colon cancer. The patient received long-term zoledronic acid treatment and has survived $>7$ years after her initial diagnosis. The patient subsequently reported bilateral hip pain that was diagnosed as osteonecrosis of the femoral head, after the presence of bone metastases was ruled out using magnetic resonance imaging. The patient underwent bilateral artificial hip joint replacements. After orthopedic surgery, the multiple distant metastases, including a brain metastasis, remained in complete remission. It is well established that BMAs, such as zoledronic acid, increase the risk of osteonecrosis of the jaw, but it is not well understood if they can increase this risk in other anatomical locations. The findings of the present case study suggested that while long-term use of BMAs may be effective in managing bone metastases, it may increase the risk of osteonecrosis in anatomical locations other than the jaw.
\end{abstract}

Correspondence to: Dr Kazuo Matsuura, Department of Breast Oncology, Saitama Medical University International Medical Center, 1397-1 Yamane, Hidaka, Saitama 350-1298, Japan

E-mail:m79kazuo@saitama-med.ac.jp

Key words: breast cancer, zoledronic acid, bilateral femoral head osteonecrosis, case report

\section{Introduction}

Bone-modifying agents (BMAs), including bisphosphonate and anti-receptor activator of NF- $\mathrm{B}$ ligand (RANKL) antibody, are effective in treating bone metastases derived from various cancer types $(1,2)$. Many oncology practice guidelines recommend combination therapy, including BMAs and anticancer agents, to treat patients with bone metastases in order to prevent skeletal-related events $(3,4)$. A main contraindication for BMAs is tooth extraction. All patients need to receive oral care prior to treatment with BMAs, as osteonecrosis of the jaw is a known adverse event of these agents (5). Conversely, incidence of osteonecrosis due to BMAs identified at other anatomical locations is not well known, and some of the evidence has been conflicting. Mont et al found that osteoporosis was not a risk factor for osteonecrosis of the femoral head (ONFH) (6), while Gangji et al reported that non-traumatic ONFH is associated with low bone mineral density (7), a disease treated mainly with BMAs. Thus, the incidence and mechanisms of ONFH in cancer patients with bone metastasis who received BMAs should be further explored. The incidence of atypical femoral fractures in elderly breast cancer patients is similar to that seen in non-breast cancer patients (8). A recent report demonstrated that zoledronate did not prevent collapse of the femoral head, and did not reduce the need for hip arthroplasty (9). Mechanisms underlying the mode of action for BMAs on the bone are still controversial $(10,11)$. Here, the case of a breast cancer patient undergoing long-term treatment with either zoledronic acid or anti-RANKL antibody, who experienced bilateral ONFH, is presented.

\section{Case report}

A 57-year-old woman, first diagnosed with stage IV cancer in 2012, had no history of heavy alcohol consumption, other comorbidities, or family history of cancer. Positron emission tomography-computed tomography (PET-CT) showed a left breast tumor with numerous metastatic tumors in the axillary lymph nodes, bone, liver, and lung (cT2N1M1) (Fig. 1). Additionally, PET-CT results suggested 
colon cancer in the sigmoid region with no regional lymph node metastasis (Fig. 2). Colonoscopy aided in the diagnosis of type 2 colon cancer (T1N0M0), and a biopsy was performed. The pathology reports revealed invasive ductal carcinoma (estrogen receptor-, progesterone receptor-, and HER2-negative) of the left breast and adenocarcinoma of the sigmoid colon (i.e., dual cancer). Considering the TN stage, the distant metastases observed upon initial diagnosis were logically assumed to be from the breast cancer and not the colon cancer. It was decided that systemic therapy for breast cancer was a priority, and if a good response was obtained, the colon cancer would be subsequently treated with surgery. The patient received six cycles of epirubicin $\left(90 \mathrm{mg} / \mathrm{m}^{2}\right)$ combined with cyclophosphamide $\left(600 \mathrm{mg} / \mathrm{m}^{2}\right)$ and zoledronic acid beginning in August 2012. Results of a follow-up PET-CT (Fig. 3) showed that the active distant metastases disappeared. The patient then underwent a laparoscopy-assisted colectomy. After the colectomy, PET-CT indicated left breast cancer with no distant metastases. The patient subsequently underwent left mastectomy with axillary lymph node dissection. Analysis of the resected tumor revealed triple-negative breast cancer. The patient received seven cycles of docetaxel $\left(75 \mathrm{mg} / \mathrm{m}^{2}\right)$ and zoledronic acid.

In August 2013, PET-CT revealed complete response. Docetaxel treatment was discontinued, but the patient continued to receive zoledronic acid after chemotherapy. In February 2014, a brain metastasis was diagnosed during BMA treatment (Fig. 4). The brain tumor was treated with 32.5 Gy of irradiation. In addition, 1-4 mg of betamethasone was administered daily for 14 days (total $=44 \mathrm{mg}$ ).

In October 2015, the patient complained of bilateral hip pain, and PET-CT suggested hip arthritis rather than relapse of bone metastases. In July 2016, she underwent magnetic resonance imaging (MRI), and was diagnosed with ONFH with no bone metastases (Fig. 5). Following this, she received bilateral artificial hip joint replacements. After the orthopedic surgery, she received zoledronic acid every 4 weeks for 3 years. Her multiple distant metastases, including brain metastasis, remain in complete remission based on PET-CT and brain MRI scans.

\section{Discussion}

Triple-negative breast cancer is difficult to treat, although chemotherapy can sometimes be effective. In this case, the patient experienced complete remission after chemotherapy, BMA treatment, and irradiation. The patient was administered zoledronic acid and has survived longer than 7 years after her initial diagnosis. This case suggests that long-term use of BMAs may result in improved control of bone metastases and support long-term survival. However, they may also induce femoral head necrosis. Although it is difficult to determine the underlying mechanisms, this case suggests to be aware regarding the occurrence of femoral necrosis in patients who receive long-term treatment with zoledronic acid. Our patient also received prophylactic corticosteroid treatment concurrent with irradiation. The total accumulated dose of betamethasone was $44 \mathrm{mg}$. Avascular necrosis of the femoral head is a rare complication related to glucocorticoid administration that has long been associated with high-dosage and/or prolonged treatment (12).
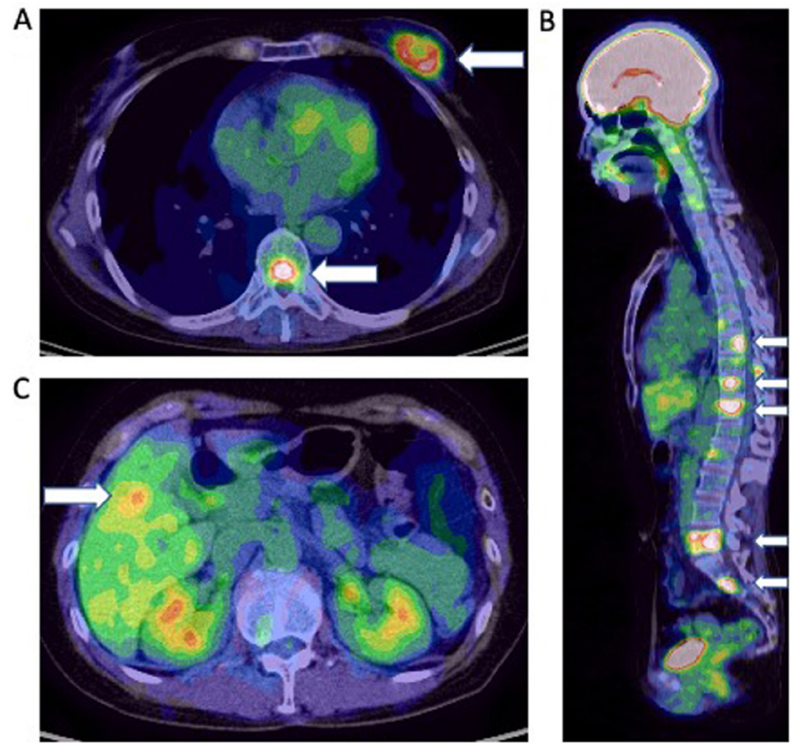

Figure 1. Left breast cancer, cT2N1M1 Stage IV. Positron emission tomography-computed tomography scans indicating (A) left breast tumor and bone metastasis, (B) multiple bone metastases and (C) liver metastasis. Arrows indicate tumors and metastases.

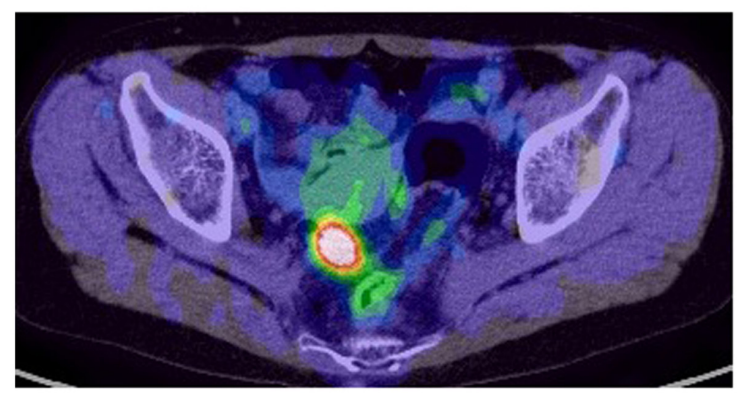

Figure 2. Colon cancer, cT1N0M0 Stage I. Positron emission tomography-computed tomography scan suggesting colon cancer in the sigmoid region with no regional lymph node metastasis.
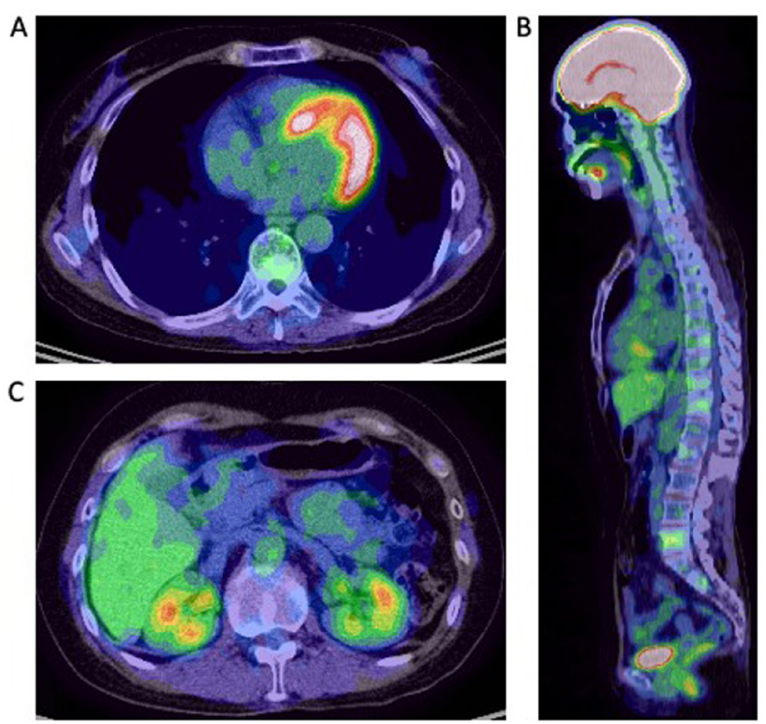

Figure 3. Post-primary systemic chemotherapy images. Positron emission tomography-computed tomography scans indicating complete responses in (A) primary breast cancer, (B) multiple bone metastases and (C) liver metastasis after systemic chemotherapy. 


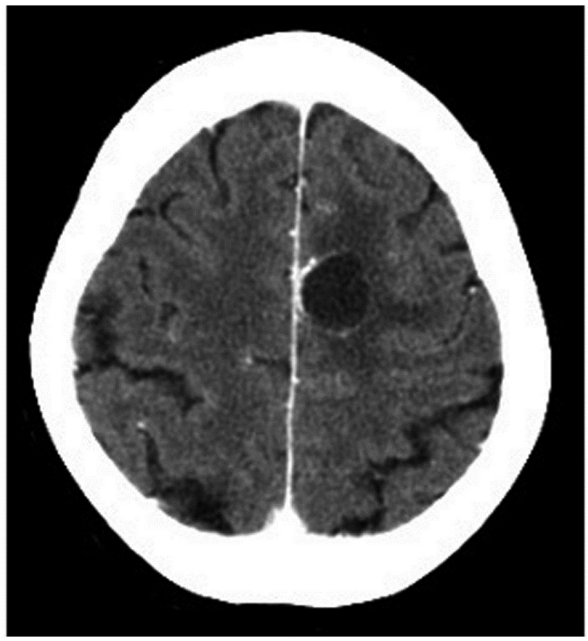

Figure 4. Brain metastasis. Computed tomography scan suggested brain metastasis in the left frontal lobe.

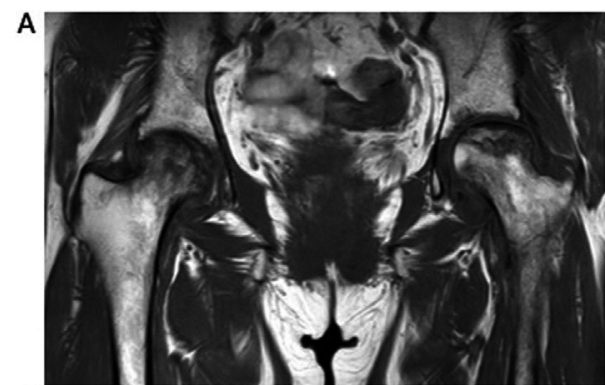

B

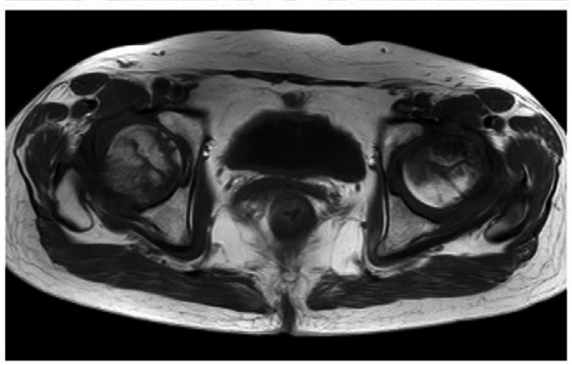

Figure 5. Bilateral hip osteonecrosis. Magnetic resonance imaging (A) corona and (B) transverse images suggested arthritis but not bone metastasis on the bilateral femoral head.

A meta-analysis by Mont et al previously reported on the relationship between corticosteroid dose and hip osteonecrosis (13). They reported that osteonecrosis incidence associated with corticosteroid treatment of $>2 \mathrm{~g}$ was $6.7 \%$ (prednisone-equivalent). Moreover, Dharmshaktu et al reported the case of a 38-year-old man who experienced ONFH after receiving $5 \mathrm{mg}$ zoledronic acid and the lowest oral dose of prednisolone. He also received $60 \mathrm{mg}$ dexamethasone in oral doses of $2 \mathrm{mg}$ for less than a month (14). In our case, the patient received $44 \mathrm{mg}$ of betamethasone with zoledronic acid, suggesting that steroids might not be the major factor in inducing ONFH. However, concurrent use of steroids and BMAs may enhance the incidence of ONFH. Lee et al reported the results of a prospective randomized trial for the prevention of femoral head osteonecrosis using zoledronic acid (9). They demonstrated that zoledronic acid was not effective in preventing femoral head necrosis. Based on basic and clinical evidence for the efficacy of bisphosphonates in improving bone strength, bisphosphonates could be a candidate for ONFH treatment. Nonetheless, we believe that long-term use of BMAs could induce ONFH because of the relationship between bisphosphonates and osteonecrosis of the jaw. Therefore, it is important in clinical practice to pay attention to chronic adverse events of BMAs. A systematic review by Bal et al revealed that bisphosphonate treatment may be prolonged for over 2 years in bone metastatic breast cancer patients with an acceptable toxicity profile (15). But the incidence of femoral head necrosis was low in that study, and not specified as one of the adverse events. Rossi et al reported a case of osteonecrosis of the distal femur associated with the use of bisphosphonates in a 74-year-old woman with metastatic breast cancer (16).

Bisphosphonates inhibit bone desorption and have therefore been used to treat osteoporosis (17). Additionally, bisphosphonates can modulate the activity of both osteoblasts and osteoclasts in metastatic bone tumors. While they can improve bone strength, they sometimes lead to bone necrosis, a severe adverse event (11). Hence, the mechanisms of bisphosphonate action on the bone remain controversial. It is also important to point out that appropriate BMA treatment regimens after complete remission need to be discussed in the development of treatment guidelines.

In conclusion, BMAs must not be discontinued until serious adverse events are recognized; however, the difference between relapse of bone metastasis and osteonecrosis caused by BMAs should be taken into consideration.

\section{Acknowledgements}

Not applicable.

\section{Funding}

No funding was received.

\section{Availability of data and materials}

The datasets used and/or analyzed during the current study are available from the corresponding author on reasonable request.

\section{Authors' contributions}

TS and KW provided the clinical data included in the text. KM wrote the manuscript draft. All authors contributed to the conception and design of the study, and interpreted and revised the PET-CT, MRI imaging and the laboratory test results. TTo and $\mathrm{TH}$ critically revised the manuscript and modified the text. TTa, AO and TTo assessed all the raw data and confirmed the authenticity of the data. All authors have read and approved the final manuscript.

\section{Ethics approval and consent to participate}

All procedures performed in the present case report were in accordance with the ethical standards of Saitama Medical University and/or national research committee and with the 1964 Declaration of Helsinki and its later amendments or 
comparable ethical standards. Written informed consent was provided by the patient included in the current case report.

\section{Patient consent for publication}

Written consent for publication was provided by the patient.

\section{Competing interests}

TS received research grants from Eisai Co., Ltd., Taiho Pharmaceutical Co., Ltd., and Chugai Pharmaceutical Co., Ltd., and received personal fees from Taiho Pharmaceutical Co. and Chugai Pharmaceutical Co. AO received research grants from AstraZeneca K.K., Eisai Co., Ltd., MSD K.K., Ono Pharmaceutical Co., Ltd., Kyowa Hakko Kirin Co., Ltd., Daiichi Sankyo Co., Ltd., Taiho Pharmaceutical Co., Ltd., Sawai Pharmaceutical Co., Ltd., Chugai Pharmaceutical Co., Ltd., Nippon Kayaku Co., Ltd., Novartis Pharma K.K., Hamamatsu Photonics K.K., Parexel International Inc., and Fuji Pharma Co. Additionally, AO received personal fees from AstraZeneca K.K., Kyowa Hakko Kirin Co., Ltd, Daiichi Sankyo Co., Ltd, Chugai Pharmaceutical Co., Ltd., and Novartis Pharma K.K. All other authors declare that they have no competing interests.

\section{References}

1. Yang M and Yu X: Management of bone metastasis with intravenous bisphosphonates in breast cancer: A systematic review and meta-analysis of dosing frequency. Support Care Cancer 28: 2533-2540, 2020.

2. Jeon HL, Oh IS, Baek YH, Yang H, Park J, Hong S and Shin JY: Zoledronic acid and skeletal-related events in patients with bone metastatic cancer or multiple myeloma. J Bone Miner Metab 38: 254-263, 2020.

3. Van Poznak C, Somerfield MR, Barlow WE, Biermann JS, Bosserman LD, Clemons MJ, Dhesy-Thind SK, Dillmon MS, Eisen A, Frank ES, et al: Role of bone-modifying agents in metastatic breast cancer: An American Society of Clinical Oncology-cancer care Ontario focused guideline update. J Clin Oncol 35: 3978-3986, 2017.

4. Cardoso F, Senkus E, Costa A, Papadopoulos E, Aapro M, André F, Harbeck N, Aguilar Lopez B, Barrios CH, Bergh J, et al: 4th ESO-ESMO International Consensus Guidelines for Advanced Breast Cancer (ABC 4). Ann Oncol 29: 1634-1657, 2018.

5. Migliorati CA, Siegel MA and Elting LS: Bisphosphonateassociated osteonecrosis: A long-term complication of bisphosphonate treatment. Lancet Oncol 7: 508-514, 2006.
6. Mont MA, Zywiel MG, Marker DR, McGrath MS and Delanois RE: The natural history of untreated asymptomatic osteonecrosis of the femoral head: A systematic literature review. J Bone Joint Surg Am Volume 92: 2165-2170, 2010.

7. Gangji V, Soyfoo MS, Heuschling A, Afzali V, Moreno-Reyes R, Rasschaert J, Gillet C, Fils JF and Hauzeur JP: Non traumatic osteonecrosis of the femoral head is associated with low bone mass. Bone 107: 88-92, 2018.

8. Takahashi M, Ozaki Y, Kizawa R, Masuda J, Sakamaki K, Kinowaki K, Umezu T, Kondoh C, Tanabe Y, Tamura N, et al: Atypical femoral fracture in patients with bone metastasis receiving denosumab therapy: A retrospective study and systematic review. BMC Cancer 19: 980, 2019.

9. Lee YK, Ha YC, Cho YJ, Suh KT, Kim SY, Won YY, Min BW, Yoon TR, Kim HJ and Koo KH: Does zoledronate prevent femoral head collapse from osteonecrosis? A prospective, randomized, open-label, multicenter study. J Bone Joint Surg Am 97: 1142-1148, 2015.

10. Russell RG, Watts NB, Ebetino FH and Rogers MJ: Mechanisms of action of bisphosphonates: Similarities and differences and their potential influence on clinical efficacy. Osteoporos Int 19: 733-759, 2008

11. Ristow O, Gerngross C, Schwaiger M, Hohlweg-Majert B, Kehl V, Jansen H, Hahnefeld L, Otto S and Pautke C: Is bone turnover of jawbone and its possible over suppression by bisphosphonates of etiologic importance in pathogenesis of bisphosphonate-related osteonecrosis? J Oral Maxillofac Surg 72: 903-910, 2014.

12. Weinstein RS: Glucocorticoid-induced osteonecrosis. Endocrine 41: 183-190, 2012.

13. Mont MA, Pivec R, Banerjee S, Issa K, Elmallah RK and Jones LC: High-dose corticosteroid use and risk of hip osteonecrosis: Meta-analysis and systematic literature review. J Arthroplast 30: 1506-1512.e5, 2015.

14. Dharmshaktu P, Aggarwal A, Dutta D and Kulshreshtha B: Bilateral femoral head avascular necrosis with a very low dose of oral corticosteroid used for panhypopituitarism. BMJ Case Rep 2016, 2016.

15. Bal O, Oksuzoglu B, Dogan M, Durnali A, Uyeturk U, Demirci A, Arslan UY, Ekinci AS, Yildirim N, Alkis N and Kilic S: Long-term outcomes of prolonged bisphosphonates more than 2 years in bone metastatic breast cancer: Risk vs benefit. Ir J Med Sci 189: 805-810, 2020.

16. Rossi L, Lascio SD, Kouros M and Pagani O: A rare avascular osteonecrosis of the knee related to bisphosphonate treatment in a patient with metastatic breast cancer. Breast Dis 35: 203-206, 2015.

17. Lyles KW, Colón-Emeric CS, Magaziner JS, Adachi JD, Pieper CF, Mautalen C, Hyldstrup L, Recknor C, Nordsletten L, Moore KA, et al: Zoledronic acid and clinical fractures and mortality after hip fracture. N Engl J Med 357: 1799-1809, 2007. 